\title{
A formulation of ALCHEMI for materials containing light elements
}

\author{
Michael G. Walls \\ Centre d'Etudes de Chimie Métallurgique, CNRS, 15 rue G. Urbain, 94407 Vitry-sur-Seine Cedex, \\ France
}

(Received November 02, 1992; accepted November 13, 1992)

\begin{abstract}
Résumé. - Une formulation multivariable de la méthode ALCHEMI est présenté dans ce travail. Celle-ci évite les problèmes dûs à la délocalisation des événements producteurs des rayons-x. La méthode détermine les distributions sur chaque site cristallin des éléments ajoutés et les facteurs de délocalisation associés à leurs signaux rayons-x, si l'on considère que les signaux des éléments de base sont localisés. Alternativement, si les signaux d'au moins un des éléments ajoutés, et de tous sauf $\mathrm{m}$ des éléments de base sont bien localisés, on peut trouver la distribution de l'impureté et le facteur de délocalisation pour l'autre élément de base. La formulation est testée avec succès sur un échantillon d'un alliage ternaire (Ni-Al-Ti) pour lequel les distribution de tous les éléments présents sont précisément connues par des mesures de diffusion anormale des rayons-x et dont un des éléments de base (Al) a un signal délocalisé.
\end{abstract}

\begin{abstract}
A multivariate formulation of the method ALCHEMI is presented, which avoids the problems arising from delocalisation of $x$-ray producing events. The method solves for the distributions of the impurity elements on the host sites, and for the delocalisation factor associated with their x-ray signals provided the host element signals can be assumed to be localised. Alternatively, if at least one impurity element's and all but one host element's signals are well localised, the impurity distribution and the delocalisation factor for the remaining host element signal can be found. The formulation is successfully tested on a ternary alloy (Ni-Al-Ti) sample for which the distributions of all the elements present are known to high accuracy from anomalous $\mathrm{x}$-ray diffraction measurements, and in which one host element $(\mathrm{Al})$ has a delocalised signal.
\end{abstract}

\section{Introduction.}

ALCHEMI [1,2] has become a standard method for the analysis of the distribution of an impurity or added element on the host sites in a crystal. Its popularity arises from its modest experimental requirements and the straightforward nature of the analysis. By comparison, other methods for obtaining the same information are less widely available, or more difficult to apply; for example anomalous $\mathrm{x}$-ray diffraction (AXD) yields high quality site distribution data, but in general requires a synchrotron source, while atom probe methods require extreme care in the preparation of the sample. For ALCHEMI, any transmission electron microscope fitted with an energy 
dispersive $x$-ray (EDX) spectrometer will suffice, and the analysis requires only the ratio of the $x$-ray signal in a channelling condition to that in a non-channelling condition for each element present. However, the standard ALCHEMI formulation assumes that for a given element, with a particular cross-section for the shell in question, the probability of $x$-ray excitation depends only on the value of the fast electron wave-function at the exact atomic position concerned, or in other words, that the excitation is completely localised. Thus a change in the channelling conditions, increasing the fast electron intensity at a particular site by a certain factor, will increase the rate of production of $x$-rays at the site by that same factor, irrespective of the atom species present or the shell considered. This assumption is known to break down for $x$-rays of energy less than about $2 \mathrm{keV}$, which can be excited by fast electrons passing within a non-negligible impact parameter $\mathrm{b}$ of the exact atom position. The wave function throughout the sphere of radius $b$ centred on the atom must then be considered, and in the case of very highly delocalised (low energy) excitations, the effects of channelling will become negligible and the x-ray intensity observed will depend only on the average value of electron intensity throughout the irradiated region. The presence of light elements, for which the only peak available in the spectrum lies in this region, can therefore render the analysis difficult [3-6].

A number of methods of correcting for these effects have been proposed, notably through the introduction of a "delocalisation factor", defined as the ratio of the observed factor by which the signal increases or decreases between two orientations to the factor which would be observed if the excitation were entirely localised. Such factors can either be extrapolated from values measured for neighbouring low-energy peaks coming from elements which also have a high energy localised peak in the spectrum [3], or theoretically estimated via calculations of the appropriate value of $b$ [5], but the results are not sufficiently general to be considered as a complete solution of the problem.

Rossouw et al. [7] realised that the conventional formulation, using ratios of the signal for an element in different spectra, was prone to error propagation, and proposed a "statistical" or multivariate approach to the analysis, in which the signal from the additional element in various orientations was plotted as a linear combination of the signals of the host species. The coefficients required to fit the data thus plotted to a straight line of unity slope yield the concentrations of all elements present and the distribution of the additional element on the host sites. However, Rossouw et al. did not treat explicitly the problem of delocalisation, but simply stated that their analysis should be less susceptible to its effects than the conventional approach. Qian et al. [8] have confirmed the utility of the multivariate approach for the analysis of ternary alloys.

In this paper a formulation is developed in which the fundamental ideas of the multivariate method are retained, the difference being that delocalisation is treated explicitly in the analysis. It is also assumed that the concentrations of the elements concerned are known from some previous measurement (e.g. a standard EDX analysis). The relevant $k$ factors [9] for the peaks concerned must in any case also be known. The multivariate plot then yields the distribution of the additional elements and their delocalisation factors, on the assumption that the host signals used are localised. Alternatively, if one or more of the impurity signals can be taken as localised, the delocalisation of one host element signal can be measured. The validity of the formulation is tested experimentally using an ordred $\mathrm{L1}_{2}$ type ternary alloy sample for which the distribution of the third element on the two distinct crystal sites has already been measured using AXD [10, 11].

\section{Alternative formulation of ALCHEMI.}

The central assumption in this method is that the signal from a delocalised excitation can be considered as being composed of two parts; a 'localised' part which is proportional to the value of the 
fast electron wave-function at the exact atomic position, and a 'delocalised' part which is proportional to the average wave-function throughout the irradiated region. Thus in a spectrum acquired for time $t$ in a fixed orientation on a fixed part of the sample, the counts $I_{X}$ for element $X$ will be given by

$$
I_{\mathrm{X}}=t K_{\mathrm{X}} n_{\mathrm{X}}\left\{\left|\psi_{\mathrm{X}}\right|^{2} L_{\mathrm{X}}+\left|\psi_{0}\right|^{2}\left(1-L_{\mathbf{X}}\right)\right\}
$$

where $K_{\mathrm{X}}=$ counts per atom $\mathrm{X}$ per unit time per unit $|\psi|^{2}, n_{\mathrm{X}}=$ number of atoms of $\mathrm{X}$ under the beam, $\left|\psi_{\mathrm{X}}\right|^{2}=$ mean fast electron intensity on sites of atom $\mathrm{X},\left|\psi_{0}\right|^{2}=$ mean fast electron intensity in the beam and $L_{X}=$ part of excitation which is localised. $\left(0<L_{\mathbf{X}}<1\right)$. This assumption, while not really justified in purely physical terms, can easily be shown to be equivalent to the assumption of the existence of a constant delocalisation factor $L_{X}$ in the standard method (see appendix) and is a hypothesis which is tested by the analysis given below. It is not the same as the assumption [7] that the wave-function consists of a channelled part and a dechannelled part. In fact it appears to be equivalent to assuming that the power spectrum of the Bloch waves present does not change significantly in going from one channelling condition to another. A more detailed investigation of this aspect of the problem is in preparation.

For clarity of notation we will treat the case of a three element sample of composition $A_{f A} B_{f B}$ $\mathrm{C}_{f \mathrm{C}}\left(\mathrm{A}, \mathrm{B}\right.$ are host elements, $\mathrm{C}$ is impurity, $\left.f_{\mathrm{A}}+f_{\mathrm{B}}+f_{\mathrm{C}}=1\right)$ in which a proportion $p_{\mathrm{CA}}$ of the atoms of $\mathrm{C}$ are on site type $\mathrm{A}$ and $p_{\mathrm{CB}}\left(=1-p_{\mathrm{CA}}\right)$ are on site type $\mathrm{B}$. We will treat separately the situation in which element $\mathrm{C}$ may have a delocalised signal, and that in which the signal from either A or B is delocalised. Extensions to cases involving more elements (host or impurity) are straightforward. We will also assume here that no anti-site defects or interstitials are present.

2.1 C DELOCALISED, A AND B LOCALiSED $\left(L_{\mathrm{A}}=L_{\mathrm{B}}=1\right)$. - Consider spectra acquired for a time $t$, at constant dose rate $\left(\left|\psi_{0}\right|^{2}=\right.$ const. $)$, fixed orientation and fixed position $\left(N_{\mathrm{at}}=\right.$ no. of atoms under the beam). The counts for the three elements will be:

$$
\begin{gathered}
I_{\mathrm{A}}=N_{\mathrm{at}} t K_{\mathrm{A}} f_{\mathrm{A}}\left|\psi_{\mathrm{A}}\right|^{2} \\
I_{\mathrm{B}}=N_{\mathrm{at}} t K_{\mathrm{B}} f_{\mathrm{B}}\left|\psi_{\mathrm{B}}\right|^{2} \\
I_{\mathrm{C}}=N_{\mathrm{at}} t K_{\mathrm{C}} f_{\mathrm{C}}\left\{p_{\mathrm{CA}}\left\{\left|\psi_{\mathrm{A}}\right|^{2} L_{\mathrm{C}}+\left|\psi_{0}\right|^{2}\left(1-L_{\mathrm{C}}\right)\right\}+\left(1-p_{\mathrm{CA}}\right)\left\{\left|\psi_{\mathrm{B}}\right|^{2} L_{\mathrm{C}}+\left|\psi_{0}\right|^{2}\left(1-L_{\mathrm{C}}\right)\right\}\right\}
\end{gathered}
$$

Note that $K_{\mathrm{C}} / K_{\mathrm{X}}$ is just $k_{\mathrm{CX}}$, the standard relative $k$ factor for the two elements at the microscope operating voltage being used (N.B. as formulated here the $k$ factors must be those used to give atomic fractions, not weight fractions). Substituting (1) and (2) into (3) we obtain

$$
I_{\mathrm{C}}=k_{\mathrm{CA}}\left(f_{\mathrm{C}} / f_{\mathrm{A}}\right) p_{\mathrm{CA}} L_{\mathrm{C}} I_{\mathrm{A}}+k_{\mathrm{CB}}\left(f_{\mathrm{C}} / f_{\mathrm{B}}\right)\left(1-p_{\mathrm{CA}}\right) L_{\mathrm{C}} I_{\mathrm{B}}+N_{\mathrm{at}} t K_{\mathrm{C}} f_{\mathrm{C}}\left|\psi_{0}\right|^{2}\left(1-L_{\mathrm{C}}\right)
$$

which can be rewritten

$$
I_{\mathrm{C}}=\alpha I_{\mathrm{A}}+\beta I_{\mathrm{B}}+\gamma
$$

where $\alpha, \beta$ and $\gamma$ are constants for all orientations, provided the irradiated volume can be kept the same. Thus if spectra are acquired at constant dose for various channelling conditions from the same area of the sample, and if we plot for each spectrum $I_{\mathrm{C}}$ on the $z$-axis against $I_{\mathrm{A}}$ along $x$ and $I_{\mathrm{B}}$ along $y$, the data should lie in a plane with slopes $\alpha$ and $\beta$ along the $x$ and $y$ axes, and intercept $\gamma$ on the $z$ axis. $\alpha, \beta$ and $\gamma$ can thus be found from a least-squares procedure, and from (4) and (5) we obtain

$$
p_{\mathrm{CA}}=\alpha^{\prime} /\left(\alpha^{\prime}+\beta^{\prime}\right)
$$




$$
L_{\mathrm{C}}=\alpha^{\prime}+\beta^{\prime}
$$

where $\alpha^{\prime}=\left(\alpha / k_{\mathrm{CA}}\right)\left(f_{\mathrm{A}} / f_{\mathrm{C}}\right)$ and $\beta^{\prime}=\left(\beta / k_{\mathrm{CB}}\right)\left(f_{\mathrm{B}} / f_{\mathrm{C}}\right)$. If we now consider the limiting cases $L_{\mathrm{C}}=0$ and $L_{\mathrm{C}}=1$ we get

$$
\text { case } L_{\mathrm{C}}=1: \quad I_{\mathrm{C}}=k_{\mathrm{CA}}\left(f_{\mathrm{C}} / f_{\mathrm{A}}\right) p_{\mathrm{CA}} I_{\mathrm{A}}+k_{\mathrm{CB}}\left(f_{\mathrm{C}} / f_{\mathrm{B}}\right)\left(1-p_{\mathrm{CA}}\right) I_{\mathrm{B}}
$$

This equation has only one unknown, $p_{\mathrm{CA}}$, and can thus be solved from a single spectrum provided it is acquired in a strong channelling condition (in a non-channelling condition $k_{\mathrm{CA}}\left(f_{\mathrm{C}} / f_{\mathrm{A}}\right)$ $I_{\mathrm{A}}=k_{\mathrm{CB}}\left(f_{\mathrm{C}} / f_{\mathrm{B}}\right) I_{\mathrm{B}}$, and there is no unique solution for $\left.p_{\mathrm{CA}}\right)$. If several spectra are plotted as described above, the resulting planefit to the data should pass through the origin. It may appear somewhat surprising that the ALCHEMI problem can be solved from a single spectrum, but since the elemental concentrations need to be known, a non-channelling spectrum will also generally be necessary. The situation is then equivalent to conventional ALCHEMI which can also be performed using such a pair of spectra.

$$
\text { case } L_{\mathrm{C}}=0: \quad I_{\mathrm{C}}=N_{\mathrm{at}} t K_{\mathrm{C}} f_{\mathrm{C}}\left|\psi_{0}\right|^{2}
$$

i.e. for complete delocalisation, the signal for $\mathrm{C}$ is just the non-channelled equivalent as expected. No information is available about $p_{\mathrm{CA}}$ and the data plane will be parallel to the $x$ and $y$ axes.

2.2 B DELOCALISED, A AND C LOCALISED $\left(L_{\mathrm{A}}=L_{\mathrm{C}}=1\right)$. - Proceeding as before we get

$$
\begin{aligned}
& I_{\mathrm{A}}=N_{\mathrm{at}} t K_{\mathrm{A}} f_{\mathrm{A}}\left|\psi_{\mathrm{A}}\right|^{2} \\
& I_{\mathrm{B}}=N_{\mathrm{at}} t K_{\mathrm{B}} f_{\mathrm{B}}\left\{\left|\psi_{\mathrm{B}}\right|^{2} L_{\mathrm{B}}+\left|\psi_{0}\right|^{2}\left(1-L_{\mathrm{B}}\right)\right\} \\
& I_{\mathrm{C}} N_{\mathrm{at}} t K_{\mathrm{C}} f_{\mathrm{C}}\left\{p_{\mathrm{CA}}\left|\psi_{\mathrm{A}}\right|^{2}+\left(1-p_{\mathrm{CA}}\right)\left|\psi_{\mathrm{B}}\right|^{2}\right\}
\end{aligned}
$$

which yields

$I_{\mathrm{C}}=k_{\mathrm{CA}}\left(f_{\mathrm{C}} / f_{\mathrm{A}}\right) p_{\mathrm{CA}} I_{\mathrm{A}}+\left(k_{\mathrm{CB}} / L_{\mathrm{B}}\right)\left(f_{\mathrm{C}} / f_{\mathrm{B}}\right)\left(1-p_{\mathrm{CA}}\right) I_{\mathrm{B}}+N_{\mathrm{at}} t K_{\mathrm{C}} f_{\mathrm{C}}\left(1-p_{\mathrm{CA}}\right)\left|\psi_{0}\right|^{2}\left(1-1 / L_{\mathrm{B}}\right)$

this is solved as before with

$$
\begin{aligned}
p_{\mathrm{CA}} & =\alpha^{\prime} \\
L_{\mathrm{B}} & =\left(1-\alpha^{\prime}\right) / \beta^{\prime}
\end{aligned}
$$

Treating the limiting cases:

$$
\text { case } L_{\mathrm{B}}=1: \quad I_{\mathrm{C}}=k_{\mathrm{CA}}\left(f_{\mathrm{C}} / f_{\mathrm{A}}\right) p_{\mathrm{CA}} I_{\mathrm{A}}+k_{\mathrm{CB}}\left(f_{\mathrm{C}} / f_{\mathrm{B}}\right)\left(1-p_{\mathrm{CA}}\right) I_{\mathrm{B}}
$$

as before.

case $L_{\mathrm{B}}=0:$ Here problems arise because of diverging terms, the $y$-slope becomes infinity and the intercept minus infinity. Physically this means that $I_{C}$ is no longer a function of $I_{B}$, which never changes. Multiplying all terms in (8) by a vanishingly small $L_{\mathrm{B}}$ we get

$$
I_{\mathrm{B}}=N_{\mathrm{at}} t K_{\mathrm{B}} f_{\mathrm{B}}\left|\psi_{0}\right|^{2}
$$


as was found before for $I_{\mathrm{C}}$. Thus no relation between $I_{\mathrm{A}}$ and $I_{\mathrm{C}}$ can be found. In the case of small but non-zero $L_{\mathrm{B}}$ the consequence is that the value of $\alpha$, and hence of $p_{\mathrm{CA}}$ will be imprecise because of the scatter in the values of $I_{C}$ as the channelling on B sites is varied.

\section{Experimental.}

In order to test the procedure, an experiment was performed on an ordered alloy, of which the stoichiometry was accurately known and the site occupations had been previously measured using the method of AXD [10]. This material, which has the composition $\mathrm{Ni}_{75} \mathrm{Al}_{11} \mathrm{Ti}_{14}$, consists essentially of an $\mathrm{Ll}_{2}$ type fcc structure resembling that of $\mathrm{Ni}_{3} \mathrm{Al}$ (in which the corner atoms are $\mathrm{Al}$ and the face atoms $\mathrm{Ni}$ ) but with some of the $\mathrm{Al}$ and $\mathrm{Ni}$ sites occupied by $\mathrm{Ti}$ atoms. The Ti is thus the 'additional' element, although it is in fact present at a higher concentration than the Al. The $\mathrm{K}$-peaks of Ti and $\mathrm{Ni}$ are both at high enough energies to be considered 'localised' but that of $\mathrm{Al}$ is not. We are thus in situation (2.2) above. The crystal cannot be aligned in such a way as to separate completely the two host element sites into different planes, but looking along any of the major poles one sees alternating planes of $100 \% \mathrm{Ni}$ sites and $50 \% \mathrm{Ni}, 50 \% \mathrm{Al}$ sites, which is a sufficient separation for channelling effects to be observed in the EDX spectra. The stoichiometry, which corresponds to $\mathrm{Ni}_{3}\left(\mathrm{Al}_{0.44} \mathrm{Ti}_{0.56}\right)$, implies either that all the $\mathrm{Ti}$ (and $\mathrm{Al}$ ) is on $\mathrm{Al}$ type sites, or that Ni anti-site defects are present, and in fact the AXD measurements show the latter to be the case. This could lead to errors in the ALCHEMI procedure, which assumes that no such defects exist. The consequences of this are examined below.

The microscope samples were prepared by spark-cutting a $3 \mathrm{~mm}$ diameter cylinder from the original ingot and slicing it into disks with a diamond saw. The disks were annealed at $723 \mathrm{~K}$ for 6 hours and then electropolished to perforation (80-20 perchloric-acetic acid mixture, $10 \mathrm{~V}$, room temperature). The microscope used was a Jeol 2000 FX fitted with a Seph Instruments Be window EDX spectrometer controlled by a Tracor computer. The sample was held in a Gatan liquid nitrogen double-tilt cold holder at about $100 \mathrm{~K}$, in order to increase the effects of channelling (reduction of Debye-Waller factor). This was also found to reduce specimen drift and contamination to unobservable levels. The microscope was operated at $80 \mathrm{kV}$ to reduce the effects of delocalisation (impact parameter $b$ is an increasing function of incident electron energy). Spectra were obtained from positions around all the 3 major poles, but by inspection the channelling effects appeared greatest around the [111] axis, so the spectra used for the analysis were all acquired at or near this orientation, with a maximum tilt off the pole of $5^{\circ}$ about each tilt axis. A grain was chosen whose [111] direction was almost normal to the foil surface, so that changes in sampled volume as a function of tilt could be considered as negligible compared with other errors in the analysis. A thickness fringe observation yielded a maximum value for the thickness of $\sim 60 \mathrm{~nm}$, and the take-off angle for a non-tilted sample was $45^{\circ}$. Consequently no absorption corrections were used in the ALCHEMI analysis. The spot-size used was $400 \mathrm{~nm}$ in diameter, and the beamconvergence about 3 mrad. A total of 14 spectra (500 second acquisitions) were obtained for use in the analysis, and the current arriving at the microscope screen was constant to within $1 \%$ throughout. A further 5 spectra were acquired in non-channelling conditions for the purposes of $k$ factor determination. (Since the stoichiometry was known [10], a normal non-channelling $\mathrm{x}$-ray spectrum gives the $k$ factors).

\section{Results.}

Figure 1 is a plot of the sum $\left(\alpha I_{\mathrm{Ni}}+\beta I_{\mathrm{Al}}\right)$ against $I_{\mathrm{Ti}}$ for all the spectra acquired around the [111] axis. Using least-squares methods, values of $\alpha$ and $\beta$ are found which most closely satisfy equation 
(8). The slope of the plot is thus equal to 1 . Also shown are the individual contributions $\alpha I_{\mathrm{Ni}}$ and $\beta I_{\mathrm{Al}}$. Each vertical group of points thus represents the data from one spectrum. It is seen that the signal from $\mathrm{Al}$ does not vary a great deal in any of the spectra. This is illustrated again in figure 2 , which is a plot of $\alpha I_{\mathrm{Ni}}$ against $\beta I_{\mathrm{Al}}$. Here we are effectively looking down on the least squares fit plane in the direction of the $z$ (i.e. Ti) axis. The lack of variation in the Al signal means that the slope of the plane in this direction (i.e. $\beta$ ) is not well defined in this case. Referring to equations (9) and (10) it can be seen that this implies that a fairly precise figure can be obtained for $p_{\mathrm{CA}}$, which depends only on $\alpha$, but that the delocalisation factor $L$, which depends on $\beta$, is not well determined.

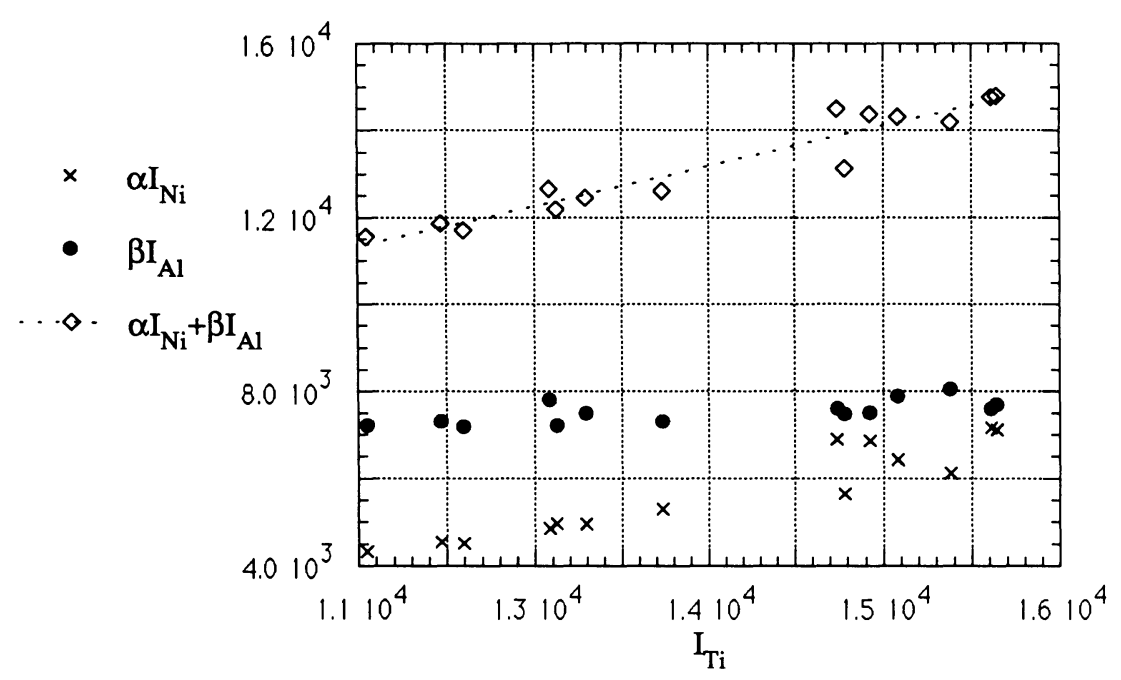

Fig. 1. - Plot of $\alpha I_{\mathrm{Ni}}+\beta I_{\mathrm{A} 1}$ against $I_{\mathrm{Ti}}$. Individual contributions $\alpha I_{\mathrm{Ni}}$ and $\beta I_{\mathrm{Al}}$ are also shown.

The numerical results of the analysis are summarised in table $\mathrm{I}$. The agreement between the values for $p_{\mathrm{CA}}$ found here and that from the method of AXD is satisfactory, and well within the limits of error for this data set. The first value given is the figure obtained assuming no anti-site defects are present. In fact, for the particular experimental case examined it was known a priori [10] that $6.7 \%$ of the Ni was actually on the Al sites and that essentially all the Al was on its own sites. Since the Al signal did not change very much as a function of orientation it can be assumed that the $\mathrm{Ni}$ atoms on the $\mathrm{Al}$ sites contributed a small and approximately constant background to the total Ni signal. This would not significantly disturb the analysis, which depends on the change in the signal from one orientation to another. However, we can also assume that the amount of $\mathrm{Ni}$ on $\mathrm{Ni}$ sites $\left(f_{\mathrm{Ni}}\right)$ is less by $6.7 \%$ than is assumed in the calculation. From equation (9) this would lead to a $6.7 \%$ overestimation of $p_{\mathrm{CA}}$, an appropriately corrected value of which is also included in table $\mathrm{I}$. This figure is in fact in slightly better agreement with the AXD measurements than the uncorrected value, but both are correct to within the experimental error limits given. These limits are estimated in a standard way from the precision to which the $k$ factors, the stoichiometry, and the signals were known. (The long counting times meant that the main source of error in the signals was not the $\sqrt{n}$ noise, but rather the variations in the calculated background subtracted by the quantification routine, depending on which parts of the spectrum were used to model it). 


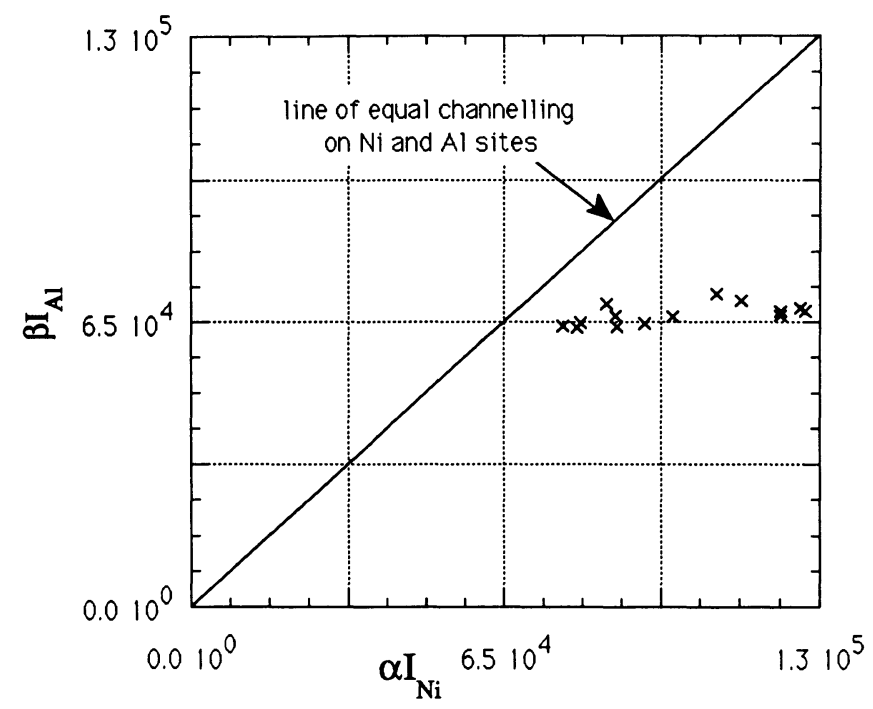

Fig. 2. $-\alpha I_{\mathrm{Ni}}$ against $\beta I_{\mathrm{Al}}$. The planefit to these points does not have a well-defined slope along the $\beta I_{\mathrm{Al}}$ axis, which means that $L$ cannot be found to high precision.

Table I. - Comparison of results obtained by various methods. The errors quoted for the AXD result are calculated from the order parameter errors quoted in [10].

\begin{tabular}{|c|c|}
\hline Experimental method & Percentage of Ti lying on Al type sites \\
\hline $\begin{array}{c}\text { ALCHEMI } \\
\text { (multivariate, no anti-site correction) }\end{array}$ & $59.7 \pm 9$ \\
\hline $\begin{array}{c}\text { ALCHEMI } \\
\text { (multivariate, with anti-site correction) }\end{array}$ & $56.1 \pm 9$ \\
\hline ALCHEMI (conventional) & $103.0 \pm 8$ \\
\hline AXD [10] & $57.1 \pm 7$ \\
\hline
\end{tabular}

The value for $L_{\mathrm{Al}}$ is somewhat smaller than values found by extrapolation methods [3, 6], although the large margin of error means that little significance can be attached to this result. The hypothesis that the data as plotted should lie in a plane (i.e., that $L$ is a constant) can be tested by calculating the value of the gamma function $Q=1-P\left((N-2) / 2, \chi^{2} / 2\right)$, i.e. the probability of obtaining a value of $\chi^{2}$ as high as or higher than that obtained in a least-squares fit procedure using $N$ spectra (for details, see for example [12]). The value obtained for this data set was $Q=0.15$, which is generally considered sufficiently high to justify the use of the hypothesis (without of course proving its validity).

It should be noted that a conventional ALCHEMI analysis on this material yielded the unphysical result that more than $100 \%$ of the $\mathrm{Ti}$ atoms were occupying $\mathrm{Al}$ sites. 


\section{Discussion.}

The formulation presented here differs from conventional ALCHEMI in that the information contained in the relative strengths of the signals from different elements is not thrown away. (The standard procedure involves taking only the ratios of signals from the same element in different channelling conditions). The price paid for using this information is the requirement that the $k$ factors and stoichiometry be known - these quantities are clearly necessary in order to render meaningful the ratios of different elements' signals. The main difference between the approach given here and that used in [7] is the introduction of a factor accounting for delocalisation effects, which in effect adds a constant to the linear combination of the host signals used to construct the plane of data (or the higher-dimensional analogue of the plane if more than two host elements are present). The formulation of [7] forces the plane to intercept the origin, and this could lead to significant errors in the presence of strong delocalisation effects.

In this particular case the effects of the presence of anti-site defects on the results are not too serious. However in general it is obvious that for the technique to be applied successfully, one needs to be able to assume that such defects are absent, or present at levels unlikely to cause significant changes in the host signals. This problem also exists of course in the conventional ALCHEMI formulation. In fact, in the case of a sample in which delocalisation effects can be assumed to be absent, a treatment similar to that developed in this paper can be used to compensate for the presence of anti-site defects. This will be given in a separate publication.

Another potential source of systematic error is the possible presence of oxides at the sample surfaces containing the three elements in a different crystallography from that in the bulk. However, if the sample thickness has not been greatly overestimated, such material should constitute less than $10 \%$ of the analysed volume. Once again such effects would also be detrimental to a conventional ALCHEMI experiment, and the choice of a region of intermediate thickness is potentially quite important in all measurements of this type.

\section{Conclusions.}

A formulation of the technique ALCHEMI has been presented which takes into account the distortions produced by delocalisation of the $x$-ray excitation events. The method has been successfully applied to the determination of the $\mathrm{Ti}$ distribution in a $\mathrm{Ni}_{3} \mathrm{Al}$ type alloy. This approach should prove applicable to a wide range of materials in which a light element such as aluminium is present, either as host or impurity.

\section{Acknowledgements.}

I am grateful to J.-P. Chevalier and P. Rez for their interest in this work, and to Y. Calvayrac for providing the sample.

\section{Appendix.}

\section{Equivalence of $L$ to delocalisation factor}

The delocalisation factor $C$ for a particular low energy EDX peak is defined as [5]:

$$
C=\left(I_{\mathrm{C}} / I_{\mathrm{R}}-1\right) /\left(I_{\mathrm{C}}^{\prime} / I_{\mathrm{R}}^{\prime}-1\right)
$$


where $I_{\mathrm{C}}$ and $I_{\mathrm{R}}$ are the measured counts in the peak concerned in a channelled and a 'random' or non-channelled spectrum respectively, and $I_{\mathrm{C}}^{\prime}$ and $I_{\mathrm{R}}^{\prime}$ are the same quantities measured for another peak from the same element which is at high enough energy to be considered localised. (eg. $I$ might refer to the Ni-L peak and $I^{\prime}$ to $\mathrm{Ni}-\mathrm{K}$ ). In the model used in this paper they can be written:

$$
\begin{aligned}
& I_{\mathrm{C}}=K\left\{\left|\psi_{\mathrm{s}}\right|^{2} L+\left|\psi_{0}\right|^{2}(1-L)\right\} \\
& I_{\mathrm{R}}=K\left|\psi_{0}\right|^{2} \\
& \mathrm{I}_{\mathrm{C}}^{\prime}=K^{\prime}\left|\psi_{\mathrm{s}}\right|^{2} \quad \text { (since } L=1 \text { for localised excitation) } \\
& \mathrm{I}_{\mathrm{R}}^{\prime}=K^{\prime}\left|\psi_{0}\right|^{2}
\end{aligned}
$$

where the constants $K, K^{\prime}$ include the $k$ factors, detection efficiency etc. and $\left|\psi_{\mathrm{s}}\right|^{2}$ and $\left|\psi_{0}\right|^{2}$ are the average fast electron densities at the atom sites and throughout the irradiated volume respectively. Thus putting $F=\left|\psi_{\mathrm{s}}\right|^{2} /\left|\psi_{0}\right|^{2}$ we obtain

$$
\begin{aligned}
C & =(F L+(1-L)-1) /(F-1) \\
& =L(F-1) /(F-1)=L
\end{aligned}
$$

\section{References}

[1] SPENCE J. and TAFTø J., J. Microscopy 130 (1983) 147.

[2] TAFTø J., J. Appl Cryst. 15 (1982) 378.

[3] BeNTLEY J., Proc. 44th EMSA (San Francisco Press, 1986) p. 704.

[4] MUNROE P.R. and BAKER I., J. Mater. Res. 6 (1991) 943.

[5] PENNYCOOK S.J., Ultramicroscopy 26 (1988) 239.

[6] MA Y. and GJøNNES J., J. Mater Res. 7 (1992) 2049.

[7] ROSSOUW C.J., TURNER P.S., WHITE T.J. and O'CONNOR A.J., Philos. Mag. Lett. 60 (1989) 225.

[8] QIAN W.D., SPENCE J.C.H., KUWABARA M. and STRYCHOR R., Script Metall. Mater. 25 (1991) 337.

[9] CLIFF G. and LORIMER G.W., Proc. Fifth Eur. Congr. Elec. Microsc. Manchester, Institute of Physics, London (1972) p. 140.

[10] MARTY A., Ph.D. Thesis (1990) Université Paris VI, France.

[11] MARTY A., Bessière M., Bley F., CALVAYRAC Y. and LeFEBVRe S., Acta Metall. Mater. 38 (1990) 345 (Describes method used. For results on alloy studied here see Ref. [10]).

[12] PRESS W.H., FLANNERY B.P., TEUKOLSKY S.A. and VETTERLING W.T., Numerical Recipes (Cambridge University Press, 1986) Chaps. 6 \& 13. 